\title{
Research component in the global alliance
}

\author{
B. JI \\ Bactériologie et Hygiène, Faculté de Médecine Pitié-Salpêtrière, \\ Paris, France
}

\begin{abstract}
During the past 15 years, tremendous progress has been made in controlling leprosy, primarily the result of the widespread implementation of multidrug therapy (MDT). Although many factors led to the success of MDT, it is fair to state that the development of the standard MDT regimens played a decisive role. The design of the regimens was based on knowledge generated by earlier research activities, including a better understanding of the threat of dapsone resistant leprosy, and the availability of newer drugs, such as rifampicin and clofazimine. The success of MDT clearly indicates that leprosy research can accelerate leprosy control.
\end{abstract}

At this moment, we are in the era of leprosy elimination. The tasks we face are far more difficult than those we have faced before. Obviously, new concepts and tools that are both more efficient and operationally less demanding are required for prevention, diagnosis and treatment of the disease, and management of leprosy reactions and nerve damage. These new concepts and tools will greatly facilitate the elimination process, and make the efforts toward elimination more sustainable. Because new concepts and tools will be generated only by research activities, whether the research is operational or laboratory-based, leprosy research must be an important component of the Global Alliance, a partnership of the World Health Organization, the International Federation of Anti-Leprosy Associations (ILEP), the Nippon Foundation, and the pharmaceutical firm, Novartis.

Like many other members of ILEP, the Association Française Raoul Follereau has a long tradition of providing financial support to research projects related to leprosy. We are particularly keen to work closely with other partners in funding leprosy research. Further to promote leprosy research, we propose the establishment of an Expert Committee on Leprosy Research, within the framework of Global Alliance. The Committee should consist of seven to nine members, with one to three members from each of the four major partners of the Alliance, the number of members being roughly proportional to the support offered leprosy research by each of the partners. The Committee should meet regularly, to identify research priorities, review research proposals, and coordinate and monitor implementation of the research projects.

Ideally, the Committee should have at its disposal funds with which to support leprosy research; otherwise, it could only function as a purely advisory body, and find it difficult to influence the setting of priorities and the directing of funds to the most meritorious and most urgent research projects. However, given that several granting agencies, such as IMMYC and THEMYC, already exist, that the US National Institutes of Health, the European Commission, the British Medical Research Council, and a number of ILEP member associations also provide support to research projects related to leprosy; and that to reach consensus among the partners on financial issues is time-consuming, it is premature to 
consider establishing a separate research fund within the Global Alliance. Instead, it may be more feasible to establish a multilateral funding mechanism, following the example of the Malawi vaccine trial and the $M$. leprae genome project. These projects clearly demonstrated that, for projects that were highly relevant, with well-prepared protocols and reasonable budgets, it was possible to secure the needed funding from multiple partners, although the process was somewhat more complicated than funding by a single agency. After a worthy project has been identified, the Expert Committee should seek funding from among the partners of the Global Alliance, including individual ILEP member associations. After additional multilaterally funded projects have succeeded, it may be possible to create a research fund within the Global Alliance.

\section{Discussion}

Dr Gupte: With respect to the funding of projects from multiple sources, will you or some one else deal with the various bureaucracies, or will it be necessary for the investigator to go from door to door?

Professor Ji: The Malawi vaccine trial was coordinated by LEPRA, and the M. leprae genome project was coordinated by the Association Française Raoul Follereau. To facilitate the funding process, I think it would be helpful to identify for each project a coordinator. If an expert committee on leprosy research should be formed within the framework of the Global Alliance, that committee could serve as coordinator.

Dr Van Brakel: It's not clear to me whether this funding process already exists, or is to be established.

Professor Ji: The Global Alliance is a very new entity. Unfortunately, the arrangement I have described is still only a proposal.

Professor Smith: My understanding is that the current situation of the Global Alliance is very uncertain. It represents only a very loose framework for collaboration. Although I think there may be merit in putting forward your proposal, I don't think we should rely on its realization. We should continue to explore other ways of securing collaboration in funding leprosy research.

Professor Britton: What is the nature of ILEP's commitment to research, and what is the role of the ILEP Medical and Social Commission. The current president and the immediate past president of the Commission are both here; can you enlighten us?

Professor Smith: The Medical and Social Commission of ILEP is a group of seven experts elected by member organizations to advise the organizations on medical and technical issues, including research. 